\title{
Impacto de uma intervenção educativa sobre Enfermagem dos Sistemas Familiares nas atitudes dos enfermeiros
}

\author{
Impact on nurses' attitudes of an educational intervention about Family Systems Nursing \\ Impacto de una interveción educativa sobre Enfermería de los Sistemas Familiares en las actitudes \\ de los enfermeros
}

\author{
Andréia Cascaes Cruz'; Margareth Angelo"
}

\begin{abstract}
RESUMO
Objetivo: avaliar o impacto de uma intervenção educativa sobre Enfermagem dos Sistemas Familiares nas atitudes dos enfermeiros. Método: pesquisa quase experimental realizada com 37 enfermeiros que atuavam no contexto neonatal/pediátrico de um hospital universitário. A etapa de intervenção, realizada em dezembro de 2014, consistiu de um treinamento com etapas teórica e prática. Os dados coletados por meio do autopreenchimento da escala "A Importância das Famílias nos Cuidados de Enfermagem - Atitudes dos Enfermeiros" foram analisados por dos testes de Kruskal-Wallis, Wilcoxon Mann-Whitney, Coeficiente de Correlação de Spearman e o teste Steel-Dwass-Critchlow-Fligner. Resultados: os enfermeiros que apresentavam escores mais baixos na fase pré-intervenção foram os que evidenciaram maior diferença positiva após, passando a apresentar escores indicativos de atitudes favoráveis à inclusão das famílias nos cuidados. Conclusão: constatou-se impacto positivo nas atitudes dos enfermeiros em relação à importância que atribuem à inclusão das famílias nos cuidados de enfermagem após a intervenção educativa.

Descritores: Enfermagem familiar; Capacitação; Atitude; Enfermeiras pediátricas.
\end{abstract}

\section{ABSTRACT}

Objective: to evaluate the impact on nurses' attitudes of an educational intervention on Family Systems Nursing. Method: quasi-experimental study with 37 nurses working in the neonatal and pediatric context of a university hospital. The intervention stage, in December 2014, consisted of theoretical and practical training. Data were collected by self-completion of the scale "The Importance of Families in Nursing Care - Nurses' Attitudes" and were analyzed by the Kruskal-Wallis, Wilcoxon Mann-Whitney, Spearman Correlation Coefficient and Steel-Dwass-Critchlow-Fligner tests. Results: nurses who scored lower in the pre-intervention phase showed greatest positive difference after the educational intervention, and returned scores indicative of attitudes favorable to the inclusion of the families in care. Conclusion: after the educational intervention, positive impact was found on nurses' attitudes regarding the inclusion of families in nursing care.

Descriptors: Family nursing; Training; Attitude; Nurses, pediatric.

\section{RESUMEN}

Objetivo: evaluar el impacto de una intervención educativa sobre Enfermería de los Sistemas Familiares en las actitudes de los enfermeros. Método: investigación casi experimental realizada junto a 37 enfermeros que trabajaban en el contexto neonatal/pediátrico de un hospital universitario. La etapa de intervención, realizada en diciembre de 2014, consistió en un entrenamiento que integró etapas teórica y práctica. Los datos recolectados por medio de instrumento llenado por el propio enfermero sobre "La Importancia de las Familias en los cuidados de Enfermería - Actitudes de los enfermeros" se analizaron por medio de las pruebas de Kruskal-Wallis, Wilcoxon Mann-Whitney, Coeficiente de Correlación de Spearman y el test Steel- Dwass-Critchlow-Fligner. Resultados: los enfermeros que presentaban escores más bajos en la fase pre-intervención fueron los que evidenciaron mayor diferencia positiva después de la intervención educativa, pasando a presentar escores indicativos de actitudes favorables a la inclusión de las familias en los cuidados. Conclusión: se constató un impacto positivo en las actitudes de los enfermeros, después de la intervención educativa, en relación a la importancia que atribuyen a la inclusión de las familias en los cuidados de enfermería. Descriptores: Enfermería de la família; Capacitación; Actitud; Enfermeras pediátricas.

\section{INTRODUÇÃO}

Enfermagem dos Sistemas Familiares é uma perspectiva de cuidado que privilegia o relacionamento estabelecido entre paciente, família e profissionais de saúde. De acordo com esta perspectiva, o sistema familiar constitui o objeto de cuidado, e este é definido pelas relações es- tabelecidas entre as partes. Os pensamentos e as atitudes dos enfermeiros devem estar centrados nas interações e na reciprocidade entre família, doença e enfermeiro ${ }^{1}$.

A abordagem de cuidado preconizada pela Enfermagem dos Sistemas Familiares requer que o profis-

'Enfermeira. Doutora em Ciências. Líder do Grupo de Estudos em Enfermagem e Família (GEENF). Universidade de São Paulo, Escola de Enfermagem, São Paulo, Brasil. E-mail: deiacascaes@gmail.com.

"Enfermeira. Doutora em Ciências. Professora Titular do Departamento de Enfermagem Materno-Infantil e Psiquiátrica. Líder do Grupo de Estudos em Enfermagem e Família (GEENF). Universidade de São Paulo, Escola de Enfermagem, São Paulo, Brasil. E-mail: angelm@usp.br. 
sional adote uma lente conceitual mais ampliada, para além do indivíduo/paciente, que focalize sua avaliação nas competências e forças familiares, e que desenvolva habilidades para realizar entrevistas com famílias, o que frequentemente torna desafiadora sua implementação na prática clínica².

O crescente interesse internacional em uma abordagem sistêmica para o atendimento dos pacientes e famílias, como a proposta pela Enfermagem dos Sistemas Familiares, exige uma atenção especial sobre como este conhecimento deve ser mobilizado para a prática clínica, o que inclui a transferência deste conhecimento para os profissionais que irão implementá-lo. A transferência do conhecimento refere-se a um processo que visa reduzir as lacunas entre o que já é conhecido cientificamente e a implementação desse conhecimento na prática, com o propósito de obter melhores resultados para pacientes e famílias, bem como, para melhorar a produtividade nos sistemas de cuidado em saúde ${ }^{3,4}$.

Pesquisas acerca da transferência do conhecimento relacionado à Enfermagem dos Sistemas Familiares vêm sendo realizadas desde o início do século XXI, em países como a Islândia e o Canadá, promovendo a circularidade entre o conhecimento teórico e a prática clínica ${ }^{5}$. Os resultados de pesquisas recentes mostraram que a tradução do conhecimento efetuada, essencialmente, por meio de intervenções educacionais baseadas nesta perspectiva, têm impacto positivo nas atitudes de enfermeiros sobre a importância que atribuem à inclusão das famílias nos cuidados $^{6-10}$, bem como na confiança, conhecimento e habilidades para entrevistar e cuidar de famílias ${ }^{8,9}$.

As atitudes englobam componentes afetivos, cognitivos e comportamentais ${ }^{11}$. A aplicabilidade da Enfermagem dos Sistemas Familiares é diretamente influenciada pelas atitudes dos enfermeiros em relação à importância que atribuem à inclusão das famílias na assistência ${ }^{10}$. Torna-se, portanto, essencial avaliar essas atitudes, pois elas constituem um pré-requisito fundamental para o enfermeiro praticar uma abordagem de cuidado que tem a família como foco, influenciando na qualidade do relacionamento estabelecido ${ }^{12,13}$.

Apesar dos resultados promissores para pacientes, famílias e enfermeiros diante da implementação da Enfermagem dos Sistemas Familiares, a transferência do conhecimento relativo a esta área do saber para a prática clínica de enfermagem é desafiadora, sobretudo porque esta abordagem, essencialmente relacional, requer o desenvolvimento de um olhar conceitual para o sistema familiar e de habilidades de entrevista familiar, que muitas vezes encontram entraves para serem efetuadas e implementadas nos contextos de prática ${ }^{2}$.

Face ao exposto, esta pesquisa teve como elemento propulsor para seu desenvolvimento o seguinte questionamento: Qual é o impacto de uma intervenção educativa sobre Enfermagem dos Sistemas Familiares nas atitudes dos enfermeiros sobre a importância que atribuem à inclusão das famílias nos cuidados de enfermagem?

Configurou-se como objetivo do estudo: Avaliar o impacto de uma intervenção educativa sobre Enfermagem dos Sistemas Familiares nas atitudes dos enfermeiros acerca da importância de incluir as famílias nos cuidados de enfermagem.

\section{MÉTODO}

Foi realizada uma pesquisa de intervenção do tipo quase experimental com desenho de série temporal interrompida com um único grupo, sem aleatoriedade, utilizando pré e pós-teste, com uma amostra de conveniência. $\mathrm{O}$ estudo foi conduzido em um Hospital Universitário (HU) localizado na cidade de São Paulo, que vêm passando por um processo revisão de suas práticas para inclusão das famílias na assistência em algumas unidades das áreas pediátricas e materno-infantis. Foram convidados para participar da pesquisa os enfermeiros que atuavam no Alojamento Conjunto, Berçário, Pediatria, Pronto Socorro Infantil, Unidade de Terapia Intensiva Neonatal e Pediátrica e residentes da área pediátrica. Foram critérios de inclusão: ser enfermeiro assistencial das referidas unidades ou residente de enfermagem da área pediátrica, ter experiência profissional mínima de seis meses, e ter interesse e disponibilidade para participar das etapas propostas pelo estudo. Foram excluídos os participantes que não completaram as etapas teórica e prática (dramatização e/ou entrevista com a família no campo) do treinamento.

\section{Intervenção educativa}

A intervenção educativa consistiu em um treinamento constituído de etapas teórica e prática com os objetivos primários de sensibilizar os enfermeiros para "pensar família" e desenvolver habilidades para realizar entrevistas com famílias. "Pensar família" é definido como ter a consciência de que os pacientes são sempre parte de uma família e, portanto, os membros da família precisam sempre ser considerados no cuidado de enfermagem ${ }^{9}$. Com base na perspectiva da Enfermagem dos Sistemas Familiares, os Modelos Calgary de Avaliação e Intervenção com a Família ${ }^{14}$ foram escolhidos para embasar o conteúdo do treinamento. A etapa teórica compreendeu uma carga horária de cinco horas e foi executada por meio de aula expositiva dialógica, discussão e debates em grupo. A etapa prática consistiu de dramatização de entrevista e/ou entrevista com famílias na prática clínica. A realização da entrevista com famílias na prática clínica era opcional, mas só poderia ser realizada pelos enfermeiros que realizaram a etapa teórica. Um instrumento denominado "Guia para Atendimento das Famílias na Prática Clínica de Enfermagem"15 , desenvolvido pelas autoras, foi utilizado na etapa prática com o objetivo nortear e registrar o processo de entrevista e colaborar com o desenvolvimento de habilidades nos enfermeiros para tal. 
A prática clínica foi planejada pautando-se nos conceitos de coaching, modelo de papel e mentoria, assim, durante duas semanas uma das pesquisadoras circulou pelas unidades assistenciais, convidando os enfermeiros que completaram a etapa teórica, para juntamente com ela realizarem a entrevista de alguma família, ou para discutir os casos com aqueles que realizaram as entrevistas sozinhos. Foram incluídos na amostra do estudo os participantes que completaram a etapa teórica e que realizaram uma ou ambas as propostas da etapa prática.

\section{Instrumento de medida}

Para avaliar as atitudes dos enfermeiros acerca da importância que atribuem à inclusão das famílias nos cuidados de enfermagem foi aplicado nas fases pré e um mês pós-intervenção o instrumento "Importância das Famílias nos Cuidados de Enfermagem - Atitudes dos Enfermeiros (IFCE-AE)"16,17. Trata-se de um instrumento de autopreenchimento, composto por 26 itens que integram três fatores ou subescalas: Fator/Subescala 1: Família - parceiro dialogante e recurso de coping (12 itens); Fator/Subescala 2: Família - recurso nos cuidados de enfermagem (10 itens); Fator/Subescala 3 Família fardo (4 itens). O escore da escala total IFCE-AE varia de 26 a 104. Escores mais altos na escala total e em todas as subescalas indicam atitudes de maior apoio à inclusão das famílias nos cuidados pelos enfermeiros.

\section{Análise dos dados}

Os dados provenientes dos instrumentos IFCE-AE foram tabulados no programa Microsoft Excel 2010, e posteriormente exportados para o banco de dados do programa Statistical Package for the Social Sciences (SPSS) 22. Foram utilizados os testes não paramétricos de Kruskal-Wallis, o teste Wilcoxon Mann-Whitney e o Coeficiente de Correlação de Spearman e o teste Steel-Dwass-Critchlow-Fligner (post hoc). Foi considerado o valor de significância de $5 \%(p \leq 0,05)$ na comparação das variáveis analisadas.

Os escores e os quartis da escala total e todas as subescalas evidenciados na fase pré-intervenção foram utilizados como nota de corte para indicar os participantes que tinham escores indicativos de atitudes que apoiavam ou não o envolvimento das famílias nos cuidados de enfermagem. Foram consideradas atitudes de apoio em relação à participação das famílias nos cuidados de enfermagem aqueles participantes que apresentaram escores entre o quartil 1 e o quartil 3 e atitudes que não apoiavam as famílias aqueles abaixo do primeiro quartil (q1) 12

\section{Aspectos éticos}

O projeto de pesquisa foi registrado sob o número 29472414.2.0000.5392 no Certificado de Apresentação para Consideração Ética (CAAE), submetido à apreciação do Comitê de Ética em Pesquisa da Escola de Enfermagem da Universidade de São Paulo e do Comitê de Ética em Pesquisa da Instituição coparticipante, sendo aprovado sob o parecer de número 657.118. Antes da coleta de dados pré-intervenção, todos os enfermeiros convidados receberam informações em relação ao estudo e aos aspectos éticos, de acordo com a Resolução do Conselho Nacional de Saúde 466/2012, aqueles que optaram por participar oficializaram sua decisão com a assinatura do Termo de Consentimento Livre e Esclarecido.

\section{RESULTADOS}

Iniciaram o treinamento 40 enfermeiros e 37 o finalizaram. Destes, 23 enfermeiros realizaram a entrevista no campo clínico, dois quais, 11 realizaram uma entrevista sozinhos, dez (10) realizaram uma entrevista juntamente com a pesquisadora e dois (2) realizaram duas entrevistas, uma sozinhos e uma acompanhados da pesquisadora.

Em relação ao preenchimento da escala IFCE-AE, 37 enfermeiros a preencheram na fase pré-intervenção e 31 na fase pós-intervenção. Das 31 escalas que retornaram na fase pós-intervenção, não foi possível encontrar o indivíduo correspondente na fase pré- intervenção em quatro deles, portanto, a amostra válida para comparação dos escores pré e pós-intervenção constituiu-se de 27 indivíduos.

A Tabela 1 revela os escores da amostra na fase pré-intervenção para a Escala IFCE-AE total e subescalas, indicando as atitudes dos enfermeiros que iniciaram o treinamento acerca da importância que atribuem às famílias nos cuidados de enfermagem e as notas de corte consideradas conforme os quartis apresentados. Para a escala total IFCE-AE, cujo escore pode variar entre 26 e 104, foi possível observar uma média de 79,89 (DP=9,422), com o escore variando entre 58 (mínimo) e 100 (máximo) dentre os respondentes. Metade deles apresentou escore inferior a 80 (abaixo da média do grupo) e $25 \%$ um escore inferior a 73,5 (quartil 1), in-

TABELA 1: Atitudes dos enfermeiros acerca da importância de incluir as famílias nos cuidados de enfermagem na fase pré-intervenção. São Paulo, SP, Brasil 2015. ( $n=37)$.

\begin{tabular}{ccccccccc}
\hline & N & Média & DP & Mínimo & Máximo & Quartil 1 & Quartil 2 & Quartil 3 \\
\hline Escala IFCE-AE (26-104)* & 37 & 79,89 & 9,422 & 58 & 100 & 73,5 & 80 & 85 \\
Subescala 1 (12-48)* & 37 & 36,16 & 4,828 & 27 & 48 & 33 & 35 \\
Subescala 2 (10-40)* & 37 & 32,22 & 3,449 & 26 & 38 & 29 & 32 \\
Subescala 3 (4-16)* & 37 & 11,51 & 2,231 & 5 & 16 & 11 & 12 \\
\hline
\end{tabular}

Nota: Subescala 1 - Família: parceiro dialogante e recurso de coping. // Subescala 2 - Família: recurso nos cuidados de enfermagem. // Subescala 3 - Família: Fardo *Possível faixa de pontuação. 
dicando que um quarto dos enfermeiros respondentes tinham atitudes que não apoiavam o envolvimento das famílias nos cuidados de enfermagem.

Na fase pré-intervenção, no que concerne a variável que se refere ao questionamento sobre o contato prévio do enfermeiro com conteúdo relacionado à Enfermagem da Família, houve diferença estatisticamente significativa para a Escala total IFCE-AE $(p=0,037)$ e para a subescala 2 - "Família: recurso nos cuidados de enfermagem" $(p=0,017)$. Constatou-se que os enfermeiros que já haviam tido contato com algum conteúdo relacionado a esta área do conhecimento apresentaram maiores médias dos escores para a escala total IFCE-AE $(81,22)$ e para a subescala 2- "Família: recurso nos cuidados de enfermagem" $(32,81)$ quando comparados àqueles que não tiveram contato com algo relacionado a esta área do conhecimento, os quais apresentaram média de 71, 40 para a escala total IFCE-AE e 28,40 para a subescala 2- "Família: recurso nos cuidados de enfermagem". Não foram evidenciadas diferenças estatisticamente significa- tivas pwara a subescala 1 - "Família: parceiro dialogante e recurso de coping" ( $p=0,064)$ e subescala 3 - "Família: Fardo" $(p=0,111)$ em relação a esta variável.

Conforme evidenciado na Tabela 2, os enfermeiros do Alojamento Conjunto, que apresentavam antes da intervenção escores indicativos de atitudes de não apoio à inclusão das famílias nos cuidados $(71,13)$, foram os que apresentaram maior diferença positiva após a intervenção, passando a apresentar uma média de escore indicativa de atitudes de apoio à inclusão das famílias nos cuidados de enfermagem.

Os enfermeiros que integraram o grupo de residentes da área pediátrica, seguido pelos enfermeiros do pronto socorro infantil, apresentaram a maior média de escore, indicativo de atitudes de apoio às famílias, tanto na fase pré quanto pós-intervenção (Tabela 2).

A realização de entrevista na prática clínica com famílias foi a variável que indicou diferença estatisticamente significativa nas atitudes dos enfermeiros quando os grupos foram comparados nas fases pré e

TABELA 2: Comparação do escore total da escala IFCE-AE e dos escores das subescalas pré e pós-intervenção com o setor em que os enfermeiros trabalham. São Paulo, SP, Brasil, 2015. $(n=37)$.

\begin{tabular}{|c|c|c|c|c|c|c|c|}
\hline \multirow[b]{2}{*}{ Setor } & \multicolumn{3}{|c|}{ Pré-intervenção } & \multicolumn{3}{|c|}{ Pós-intervenção } & \multirow[b]{2}{*}{ Valor-p* } \\
\hline & $\mathrm{N}$ & Média & DP & $\mathrm{N}$ & Média & DP & \\
\hline & & Escala IFCE-AE & & & scala IFCE-AE & & 0,068 \\
\hline$A C$ & 8 & 71,13 & 7,75 & 6 & 79,17 & 9,09 & \\
\hline BER & 9 & 81,22 & 5,02 & 7 & 78,43 & 5,26 & \\
\hline PED & 6 & 82,50 & 7,23 & 5 & 83,00 & 4,06 & \\
\hline PSI & 3 & 83,33 & 1,53 & 3 & 83,67 & 6,66 & \\
\hline RES & 5 & 89,60 & 9,53 & 3 & 93,00 & 5,20 & \\
\hline UTIPN & 6 & 77,17 & 12,01 & 3 & 79,67 & 14,36 & \\
\hline \multirow[t]{2}{*}{ Total } & 37 & 79,89 & 9,42 & 27 & 81,78 & 8,11 & \\
\hline & & Subescala 1 & & & Subescala 1 & & 0,060 \\
\hline$A C$ & 8 & 31,75 & 3,37 & 6 & 36,00 & 5,02 & \\
\hline BER & 9 & 36,22 & 2,59 & 7 & 34,71 & 2,50 & \\
\hline PED & 6 & 37,67 & 3,67 & 5 & 37,60 & 2,61 & \\
\hline PSI & 3 & 37,33 & 1,53 & 3 & 39,33 & 4,04 & \\
\hline RES & 5 & 41,60 & 4,72 & 3 & 42,33 & 3,79 & \\
\hline UTIPN & 6 & 35,33 & 6,62 & 3 & 38,00 & 7,00 & \\
\hline \multirow[t]{2}{*}{ Total } & 37 & 36,16 & 4,83 & 27 & 37,26 & 4,37 & \\
\hline & & Subescala 2 & & & Subescala 2 & & 0,098 \\
\hline$A C$ & 8 & 29,25 & 2,38 & 6 & 31,17 & 3,60 & \\
\hline BER & 9 & 32,89 & 1,76 & 7 & 31,43 & 2,30 & \\
\hline PED & 6 & 34,00 & 2,76 & 5 & 33,40 & 2,41 & \\
\hline PSI & 3 & 34,00 & 1,00 & 3 & 32,33 & 1,53 & \\
\hline RES & 5 & 34,80 & 3,77 & 3 & 36,67 & 0,58 & \\
\hline UTIPN & 6 & 30,33 & 4,68 & 3 & 31,00 & 5,57 & \\
\hline \multirow[t]{2}{*}{ Total } & 37 & 32,22 & 3,45 & 27 & 32,37 & 3,21 & \\
\hline & & Subescala 3 & & & Subescala 3 & & 0,115 \\
\hline$A C$ & 8 & 10,13 & 2,53 & 6 & 12,00 & 0,89 & \\
\hline BER & 9 & 12,11 & 1,62 & 7 & 12,29 & 0,76 & \\
\hline PED & 6 & 10,83 & 2,93 & 5 & 12,00 & 1,22 & \\
\hline PSI & 3 & 12,00 & 1,00 & 3 & 12,00 & 1,73 & \\
\hline RES & 5 & 13,20 & 2,59 & 3 & 14,00 & 1,73 & \\
\hline UTIPN & 6 & 11,50 & 1,05 & 3 & 10,67 & 2,52 & \\
\hline Total & 37 & 11,51 & 2,23 & 27 & 12,15 & 1,46 & \\
\hline
\end{tabular}

Nota: Alojamento Conjunto (AC); Berçário (BER); Pediatria (PED); Pronto Socorro Infantil (PSI); Residentes de Pediatria (RES); Unidade de Terapia Intensiva Neonatal e Pediátrica (UTINP). 
pós-intervenção. Esta diferença se deu na Escala total IFCE-AE $(p=0,023)$ e na Subescala 1 - Família: parceiro dialogante e recurso de coping $(p=0,005)$, conforme consta na Tabela 3.

No que se refere à Escala total IFCE-AE, aqueles enfermeiros que não realizaram a entrevista com alguma família na prática clínica apresentaram uma diferença maior (6,10 para mais) entre a distribuição dos escores nas fases pré $(74,10)$ e pós intervenção $(80,20)$ do que aqueles que realizaram a entrevista na prática, os quais praticamente não apresentaram diferença ( 0,82 para menos) nas fases pré $(83,53)$ e pós $(82,71)$ intervenção (Tabela 3$)$.

Em relação à subescala 1 - Família: parceiro dialogante e recurso de coping, aqueles enfermeiros que não realizaram a entrevista na prática tiveram uma maior diferença $(3,90)$ na distribuição dos escores préintervenção $(33,20)$ e pós-intervenção $(37,10)$ do que aqueles que realizaram a entrevista no campo clínico, os quais praticamente não apresentaram diferença $(0,59$ para menos) entre as fases pré $(37,94)$ e pós $(37,35)$ intervenção (Tabela 3).

Conforme a Tabela 3, considerando apenas a fase pré-intervenção, e àqueles que preencheram à escala IFCE-AE nas fases pré e pós-intervenção, os enfermeiros que realizaram a entrevista na prática clínica apresentavam na fase pré-intervenção escores superiores àqueles enfermeiros que não realizaram a etapa de entrevista com famílias prática clínica. Para a escala total IFCE-AE àqueles que realizaram a entrevista na prática clínica tiveram um escore médio de 83,53 (DP=9,321) na fase pré-intervenção, enquanto aqueles que não aplicaram apresentaram média de 74,10 (DP=7,608). Assim, os enfermeiros que realizaram a entrevista na prática clínica na fase pós-intervenção iniciaram o treinamento com escores superiores àqueles enfermeiros que não realizaram a etapa de entrevista com famílias prática clínica.

\section{DISCUSSÃO}

Em relação às atitudes dos enfermeiros sobre a importância que atribuem à inclusão das famílias nos cuidados de enfermagem, houve impacto positivo nas atitudes dos enfermeiros após a intervenção educativa. O principal impacto ocorreu naqueles enfermeiros que apresentaram escores mais baixos na fase pré-intervenção, e que não se dispuseram a realizar a entrevista na prática clínica. Importante enfatizar que os enfermeiros que se propuseram a realizar a etapa prática no campo clínico, estavam mais abertos e disponíveis para interagir e se relacionar com as famílias, pois apresentaram escores mais elevados na fase pré-intervenção do que aqueles que optaram por não realizar a etapa prática no campo, os quais tiveram escores baixos, indicativos de atitudes não apoiadoras à participação das famílias. Acredita-se que os enfermeiros que realizaram o

TABELA 3: Comparação do escore total da escala IFCE-AE e dos escores das subescalas pré e pós-intervenção com o grupo de enfermeiros que participou ou não da entrevista na prática clínica. São Paulo, SP, Brasil, 2015. (n=27)

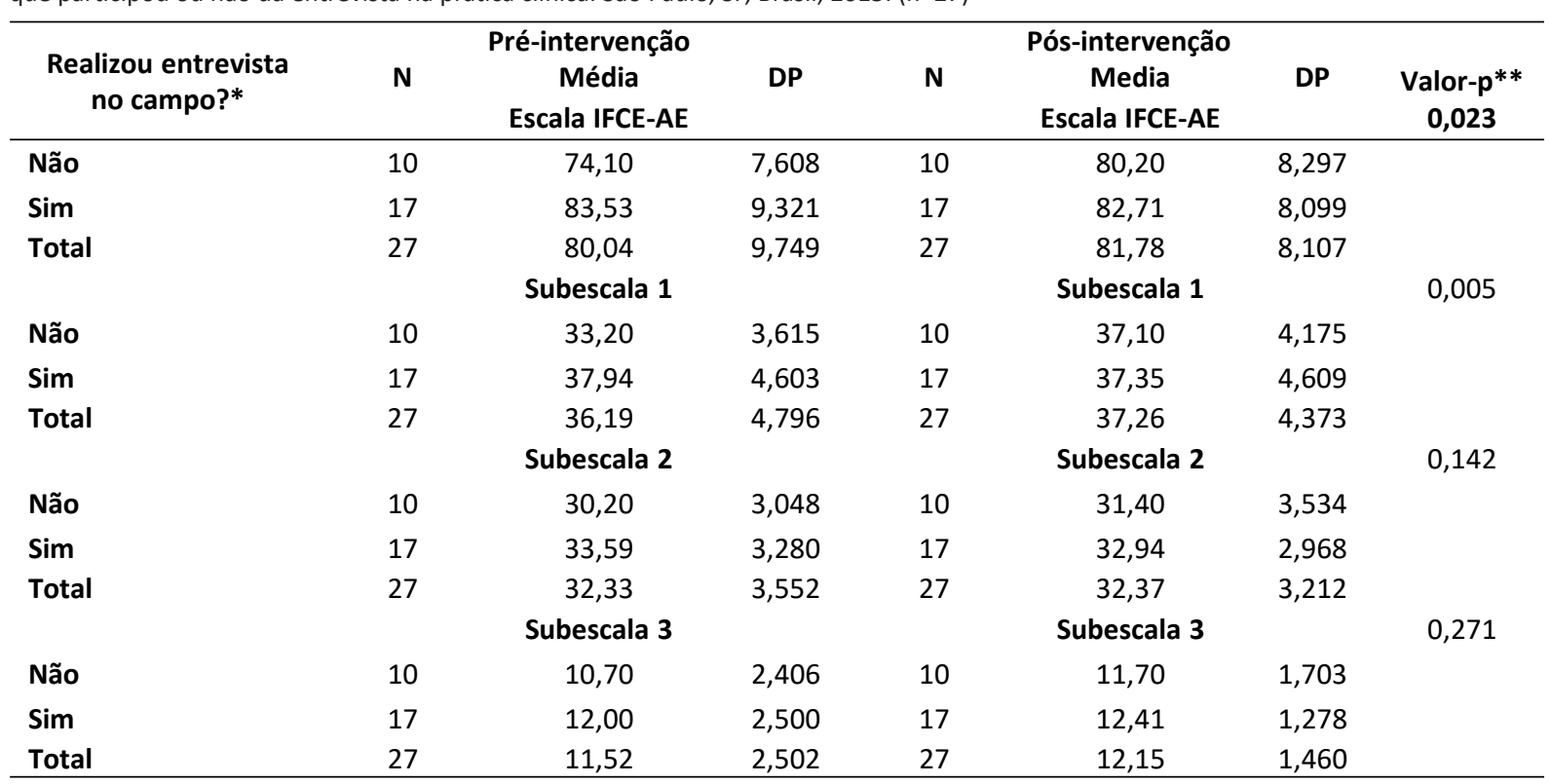

Nota: *Realizou entrevista e aplicou o guia proposto com alguma família na sua unidade?

Subescala 1 - Família: parceiro dialogante e recurso de coping. //Subescala 2 - Família: recurso nos cuidados de enfermagem. // Subescala 3 - Família: Fardo.

** Teste Wilcoxon Mann- Whitney para diferença pré e pós-intervenção.

* Os resultados apresentados na tabela sobre a realização da entrevista na Prática Clínica pelos enfermeiros, referem-se apenas à amostra que respondeu à Escala IFCE-AE nas fases pré e pós-intervenção. Estes dados não refletem o número real de enfermeiros que realizou ou não a etapa prática de entrevista no campo clínico na fase de intervenção. 
atendimento na prática clínica, por já terem escores elevados na fase pré-intervenção, não apresentaram diferença da fase pré para a fase pós-intervenção, pois já apresentavam atitudes de apoio às famílias.

Tal evidência é relevante e endossada pela literatura, pois quando se almeja a transferência do conhecimento em direção à inclusão das famílias para o contexto assistencial, atitudes de apoio dos enfermeiros à participação das famílias nos cuidados de enfermagem promovem um ambiente de cuidado em saúde que facilitam a implementação da enfermagem da família, pois atitudes positivas são uma premissa importante para o enfermeiro se abrir, incluir as famílias nos cuidados e se relacionar com elas ${ }^{12}$.

As atitudes dos enfermeiros acerca da importância de incluir as famílias nos cuidados de enfermagem já haviam sido objeto de estudo brasileiro ${ }^{17}$ realizado no mesmo local, nas mesmas unidades e utilizando o mesmo instrumento que o presente estudo, o qual indicou uma média de escore da escala total IFCE-AE de 82 , maior do que a fase pré-intervenção deste estudo, cujo escore inicial para a escala total IFCE-AE foi de 79,8. A média dos escores apresentados na escala IFCE-AE do presente estudo, foi semelhante a de um estudo conduzido em Portugal, cuja pontuação média dos enfermeiros da pediatria foi de $79,9^{18}$, e inferiores aos apresentados por outros dois estudos realizados com enfermeiras europeias ${ }^{12,19}$ que evidenciaram uma média do escore para a escala total IFCE-AE igual a 88.

Estudo sueco ${ }^{12}$ mostrou um escore correspondente ao primeiro quartil da escala IFCE-AE de 81, sendo consideravelmente maior que nos estudos brasileiros, sobretudo na fase pré-intervenção do presente estudo, que foi igual a 73,5 . No estudo brasileiro realizado anteriormente a este ${ }^{12}$, o primeiro quartil era igual a 77, isto é, passados três anos e meio entre uma avaliação e outra, a média do escore de $25 \%$ dos enfermeiros caiu consideravelmente, evidenciando o aspecto dinâmico do conceito analisado.

A avaliação das atitudes dos enfermeiros acerca da importância das famílias nos cuidados de enfermagem em relação a variável setor na fase pré-intervenção do presente estudo, assim como no estudo brasileiro prévio ${ }^{17}$, indicou diferença estatisticamente significativa para a escala IFCE-AE total e para subescala 2 - "Família: recurso nos cuidados de enfermagem". Os enfermeiros do Alojamento Conjunto foram os que apresentaram a menor média de escore dentre todos os outros setores avaliados, tanto para a escala total IFCE-AE, quanto para a subescala 2: "Família: recurso nos cuidados de enfermagem". A média do escore dos enfermeiros do Alojamento Conjunto para a escala total IFCE-AE no estudo anterior ${ }^{17}$ era de 76 , maior do que na fase pré-intervenção do nosso estudo $(71,1)$ e menor do que a fase pós-intervenção $(79,1)$. Para a subescala 2: "Família: recurso nos cuidados de enfermagem" a média do escore no primeiro estudo era de 30 , sendo menor na fase pré-intervenção deste estudo, cuja média foi de 29,2 . Estudo português encontrou uma média de 30,6 para esta subescala ${ }^{18}$.

Os enfermeiros do Pronto Socorro Infantil, que participaram de ambos os estudos nacionais, continuam apresentando escores mais elevados quando comparados aos outros grupos. No primeiro estudo nacional ${ }^{17}$ os enfermeiros do Pronto Socorro Infantil apresentavam uma média de escore igual a 87 para a escala IFCE-AE total e 35 para a subescala 2: "Família: recurso nos cuidados de enfermagem", enquanto, neste estudo, apresentaram uma média de escore pré-intervenção igual a 83,3 para a escala IFCE-AE total e 34 para a subescala 2: "Família: recurso nos cuidados de enfermagem", não apresentando diferença estatisticamente significativa na fase pós-intervenção.

Em relação a variável que questionou sobre o contato prévio do enfermeiro com conteúdo relacionado à Enfermagem da Família, houve diferença estatisticamente significativa para a Escala total IFCE-AE e para a subescala 2 - "Família: recurso nos cuidados de enfermagem", onde os enfermeiros que haviam tido contato com algum conteúdo relacionado a esta área do conhecimento apresentaram escores mais altos na fase pré-intervenção deste estudo e no estudo brasileiro anterior, indicativos de atitudes de maior apoio à inclusão das famílias nos cuidados $^{17}$. Isto mostra que a aproximação do enfermeiro com conteúdos relacionados a esta temática o torna mais sensível e aberto para as famílias.

Em contexto europeu, estudo encontrou associação significativa entre o nível educacional do enfermeiro e os escores apresentados, onde aqueles com maior nível educacional apresentaram escores mais elevados, indicando atitudes mais positivas em relação à participação das famílias nos cuidados de enfermagem. Foi encontrada ainda associação entre a idade dos enfermeiros e os escores, onde os mais jovens tinham atitudes menos positivas em termos de perceber a família como um parceiro dialogante e mais frequentemente percebiam as famílias dos pacientes como um fardo ${ }^{19}$. Diferente do estudo europeu, estas associações não foram estatisticamente significativas no presente estudo. Contudo, os enfermeiros residentes eram os mais jovens no presente estudo, e foram os que apresentaram os maiores escores neste estudo indicando que tinham atitudes mais favoráveis face às famílias do que os demais.

Estudo de intervenção educativa pautada na Enfermagem dos Sistemas Familiares realizado com enfermeiros de uma unidade psiquiátrica na Islândia ${ }^{10}$ indicou uma média de escore inicial (pré-intervenção) igual a 91,2 entre os enfermeiros do sexo feminino e 86,5 dentre os do sexo masculino, sendo consideravelmente maior que a média inicial do nosso estudo, a qual foi igual a 79,8. Na fase pós-intervenção do estudo islandês, encontrou-se diferença estatisticamente significativa apenas para a su- 
bescala 3: Família fardo, onde os escores pós-intervenção educativa indicaram que os enfermeiros tiveram redução na suas percepções acerca da família como um fardo depois de terem realizado o treinamento, o que não foi evidenciado em nosso estudo. Estudo semelhante, realizado em unidades cirúrgicas do mesmo hospital, não encontrou diferenças nas atitudes dos enfermeiros antes e após a intervenção educativa ${ }^{7}$.

O curto espaço de tempo entre a intervenção e a coleta de dados pós-intervenção, e a não realização da entrevista no campo clínico pela totalidade dos enfermeiros, foram limitações do estudo. Outra limitação pode ser atribuída ao fato do estudo ter sido realizado em uma única instituição; aplicar a intervenção em outras instituições poderia produzir resultados distintos, sobretudo, em função da cultura organizacional. Concordamos que mais pesquisas são necessárias para explorar as diferenças culturais e regionais das atitudes dos enfermeiros em relação ao seu papel e atitudes de envolvimento das famílias nos cuidados de enfermagem ${ }^{19}$.

\section{CONCLUSÃo}

Constatou-se impacto positivo nas atitudes dos enfermeiros em relação a importância que atribuem à inclusão das famílias nos cuidados de enfermagem após a intervenção educativa sobre a Enfermagem dos Sistemas Familiares que integrou etapas teórica e prática. Os enfermeiros que apresentavam atitudes de menor apoio à inclusão das famílias nos cuidados na fase pré-intervenção, foram os que apresentaram aumento significativo dos valores de escore indicativos de atitudes de suporte às famílias após o treinamento, no entanto, foram os que não realizaram a entrevista na prática clínica com as famílias.

Os enfermeiros que possuíam escores mais elevados na fase pré-intervenção, ou seja, que tinham atitudes que apoiavam a inclusão das famílias nos cuidados de enfermagem, foram os que se dispuseram a realizar a etapa prática no campo clínico, indicando que atitudes positivas dos enfermeiros em relação às famílias facilitam o processo de transferência do conhecimento para a prática clínica.

As ações bem-sucedidas apresentadas neste estudo, por meio da consolidação desse processo sistemático de capacitação dos enfermeiros para o cuidado da família, podem ser reproduzidas em outras instituições, acadêmicas ou assistenciais, e assim contribuir com o avanço do conhecimento e instrumentalização de enfermeiros para atenderem famílias na prática clínica.

\section{REFERÊNCIAS}

1. Bell JM. Family systems nursing: Re-examined. J Fam Nurs [Internet]. 2009 [cited 2018 Apr 18]; 15(2):123-9. Available from: http://journals.sagepub.com/doi/10.1177/1074840709335533. 2. Duhamel F. Translating Knowledge From a Family Systems Approach to Clinical Practice: Insights From Knowledge
Translation Research Experiences. J Fam Nurs [Internet]. 4 de novembro de 2017;23(4):461-87. Available at: https://doi. org/10.1177/1074840717739030.

3. Graham ID, Logan J, Harrison MB, Straus SE, Tetroe J, Caswell W, et al. Lost in knowledge translation: Time for a map? J Contin Educ Health Prof [Internet]. 2006;26(1):13-24. Available at: http:// content.wkhealth.com/linkback/openurl?sid=WKPTLP:landingpa ge\&an=00005141-200626010-00003

4. Svavarsdottir EK, Tryggvadottir GB, Sigurdardottir AO. Knowledge Translation in Family Nursing: Does a Short-Term Therapeutic Conversation Intervention Benefit Families of Children and Adolescents in a Hospital Setting? Findings From the Landspitali University Hospital Family Nursing Implementation Project. J Fam Nurs. 2012;18(3):303-27.

5. Leahey M, Svavarsdottir EK. Implementing Family Nursing: How Do We Translate Knowledge Into Clinical Practice? J Fam Nurs [Internet]. 25 de novembro de 2009;15(4):445-60. Available at: http://journals.sagepub.com/doi/10.1177/1074840709349070 6. Svavarsdottir EK, Sigurdardottir AO, Konradsdottir E, Stefansdottir A, Sveinbjarnardottir EK, Ketilsdottir A, et al. The Process of Translating Family Nursing Knowledge Into Clinical Practice. J Nurs Scholarsh [Internet]. janeiro de 2015;47(1):5-15. Available at: http://doi.wiley.com/10.1111/jnu.12108

7. Blöndal $K$, Zoëga $S$, Hafsteinsdottir JE, Olafsdottir OA, Thorvardardottir $A B$, Hafsteinsdottir $S A$, et al. Attitudes of Registered and Licensed Practical Nurses About the Importance of Families in Surgical Hospital Units. J Fam Nurs [Internet]. 15 de agosto de 2014;20(3):355-75. Available at: http://journals.sagepub.com/ doi/10.1177/1074840714542875

8. Eggenberger SK, Sanders M. A family nursing educational intervention supports nurses and families in an adult intensive care unit. Aust Crit Care [Internet]. novembro de 2016;29(4):217-23. Available at: https://linkinghub.elsevier.com/retrieve/pii/ S1036731416300819

9. Broekema S, Luttik MLA, Steggerda GE, Paans W, Roodbol PF. Measuring Change in Nurses' Perceptions About Family Nursing Competency Following a 6-Day Educational Intervention. J Fam Nurs [Internet]. 19 de novembro de 2018;107484071881214. Available at: http://journals.sagepub. com/doi/10.1177/1074840718812145

10. Sveinbjarnardottir EK, Svavarsdottir EK, Saveman BI. Nurses attitudes towards the importance of families in psychiatric care following an educational and training intervention program. J Psychiatr Ment Health Nurs. 2011;18(10):895-903.

11.Altmann TK. Attitude: A Concept Analysis. Nurs Forum [Internet]. julho de 2008;43(3):144-50. Available at: http://doi.wiley. com/10.1111/j.1744-6198.2008.00106.x

12. Benzein E, Johansson P, Årestedt KF, Saveman B-I. Nurses' Attitudes About the Importance of Families in Nursing Care. J Fam Nurs [Internet]. 7 de maio de 2008;14(2):162-80. Available at: http://journals.sagepub.com/doi/10.1177/1074840708317058 13. Saveman B-I. Family Nursing Research for Practice: The Swedish Perspective. J Fam Nurs [Internet]. 9 de fevereiro de 2010;16(1):26-44. Available at: http://journals.sagepub.com/ doi/10.1177/1074840709360314

14. Wright LM, Leahey M. Nurses and families: A guide to family assessment and intervention. 6th ed. Philadelphia: F.A Davis Company; 2012.

15. Santos LG dos, Cruz AC, Mekitarian FFP, Angelo M. Family interview guide: strategy to develop skills in novice nurses. Rev bras enferm [Internet]. dezembro de 2017;70(6):1129-36. Available at: http://www.scielo.br/scielo.php?script=sci_arttext\&pid=S0034$-71672017000601129 \& \operatorname{lng}=e n \& \operatorname{lng}=e n$

16. Oliveira P da CM, Fernandes HIV, Vilar AISP, Figueiredo MH de JS, Ferreira MMSRS, Martinho MJCM, et al. Attitudes of nurses towards families: Validation of the scale Families' Importance in Nursing Care - Nurses Attitudes. Rev Esc Enferm. USP. 2011;45(6):1331-7. 17. Angelo M, Cruz AC, Mekitarian FFP, Santos CC da S dos, Mar- 
tinho MJCM, Martins MMFP da S. Nurses' attitudes regarding the importance of families in pediatric nursing care. Rev. Esc. Enferm. USP [Internet]. agosto de 2014;48(spe):74-9. Available at: http://www.scielo.br/scielo.php?script=sci_arttext\&pid=S0080$-62342014000700074 \&$ Ing $=e n \&$ tIng $=e n$

18. Fernandes CS, Gomes JAP, Martins MM, Gomes BP, Gonçalves LHT. The Importance of Families in Nursing Care: Nurses' Attitudes in the Hospital Environment. Rev. Enferm. Ref. [Internet]. 2015; IV
Série(7):21-30. Available at: http://esenfc.pt/rr/index.php?modu le=rr\&target=publicationDetails\&pesquisa=\&id_artigo=2547\&id revista $=24 \& i d \_$edicao $=88$

19. Luttik M, Goossens E, Ågren S, Jaarsma T, Mårtensson J, Thompson $\mathrm{D}$, et al. Attitudes of nurses towards family involvement in the care for patients with cardiovascular diseases. Eur J Cardiovasc Nurs [Internet]. 2017;16(4):299-308. Available at: http://journals.sagepub.com/doi/ 10.1177/1474515116663143 